\title{
Leprosy elimination campaign in a metropolitan leprosy project, Bombay, India
}

\author{
C. R. REVANKAR, M. S. ANTONY SAMY, \\ H. O. BULCHAND \& R. GANAPATI \\ Bombay Leprosy Project, 11 VN Purav Marg, Chunabhatti, Bombay \\ 400022, India
}

Accepted for publication 30 August 1999

\section{Introduction}

The global strategy for eliminating leprosy as a public health problem by the end of this century is primarily dependent on detection of untreated hidden leprosy patients, especially those with leprosy of consequence, and curing them with MDT. ${ }^{1}$ To unearth such cases rapidly, WHO recommended a leprosy elimination campaign (LEC) through community involvement. ${ }^{1}$ The National Leprosy Eradication Programme (NLEP) India initiated a country-wide LEC during 1998 after the initial experience in Tamil Nadu in 1997. Bombay Leprosy Project (BLP) undertook LEC in its entire project area as a part of the statewide campaign in Maharashtra State between 30 January and 5 February 1998 to identify hidden cases and to locate endemic pockets.

\section{Materials and methods}

Bombay Leprosy Project, a field research project, initiated leprosy control activities in 1976 in a well defined area with a population of 1.8 million, $50 \%$ of which is reported to be living in small and large slums. Though the whole population was brought under the LEC, more emphasis was placed on slum areas, as slum dwellers are easily accessible for physical examination and also the endemicity of leprosy is higher compared to a non-slum population. We had to follow the guidelines issued by the NLEP India through the District Leprosy Officer of Bombay to recruit volunteers (searchers), and to carry out public education, training, recording of family survey data and reporting. Two volunteers were expected to screen approximately 500 people per day. On that basis, 748 volunteers from both urban primary health care units (health posts) and the community, including college students who 
Table 1. Screening by community volunteers (\%)

\begin{tabular}{lc}
\hline Enumerated population & $1,330,372(69)$ \\
Examined population & 923,004 \\
Suspected cases & 2285 \\
Confirmed leprosy cases & $97(4)$ \\
Skin smear positive MB cases & $9(9)$ \\
Detection rate/100,000 & 10.5 \\
Smear positive case rate/100,000 & 0.97 \\
Proportion of disabled cases & $4(4)$ \\
\end{tabular}

volunteered, were selected. They were paid 40 rupees 40 (approximately $1 \$$ US) per day for one day of training and 7 days fieldwork.

All the searchers were subjected to a 1-day orientation in identif ying leprosy by looking for any pale skin patch, shiny, red skin of face and ears, hand deformities and plantar ulcers. More emphasis was placed on suspecting lepromatous leprosy. This was done by using a colour diagnostic card and demonstrating leprosy patients. They were given a list of questions on signs and symptoms of leprosy to be asked while going from house to house. They were oriented in filling in family survey forms. However, they were told to suspect all skin disease patients whose appearance suggested leprosy without performing sensory testing and neurological examination, as the objective was not to develop the diagnostic skills of the volunteers. All these volunteers worked under the supervision of trained paramedical workers. After the training, the searchers went round the slums from house to house showing the colour picture card and asking the slum dwellers questions on signs and symptoms of leprosy for 7 days (Table 1). The trained and experienced doctors of the project later confirmed the suspected cases.

Before the actual case detection, intensive community awareness programmes were carried out not only by the project staff but also by the State Health authorities through radio, TV and the press, as well as through the display of posters and banners.

\section{Results and discussion}

Of the 1.8 million control area population of BLP, the searchers were able to enlist $1,330,372$ $(74 \%)$ individuals. Of this enlisted population, 923,004 (69\%) residents were screened for leprosy within 7 days. The field staff of the project confirmed 97 (4.2\%) new patients out of the 2285 suspected individuals. As these volunteers were not trained either to test sensory impairment in the skin lesions, or to palpate the nerves and do the skin smears for acid fast bacilli, a large number of suspected cases turned out not to be leprosy, as the sensitivity and specificity was low in this campaign. The most important outcome of this campaign was the identification of 14 (14\%) MB leprosy cases of consequence, of whom nine were smear positive patients who would have remained undetected and untreated for a long time to come but for this leprosy elimination campaign. Fifty-three (53\%) were single skin lesion (SSL) PB type and 30 (31\%) were of two to five lesions PB type, while four patients had disability. The new case detection rate was $10 \cdot 5$ per 100,000 population. It is expected that the case detection rate will show a declining trend after the LEC in a given area provided such campaigns are 
Table 2. Impact of LEC on new case detection (March to December)

\begin{tabular}{|c|c|c|c|c|}
\hline \multirow[b]{2}{*}{ Type of leprosy } & \multicolumn{2}{|c|}{1998 (post-LEC) } & \multirow[b]{2}{*}{ Number } & \multirow[b]{2}{*}{$\%$} \\
\hline & Number & $\%$ & & \\
\hline MB smear positive & 43 & 25 & 19 & 15 \\
\hline MB smear negative & 13 & 7 & 22 & 17 \\
\hline $2-5$ lesions $\mathrm{PB}$ & 64 & 37 & 49 & 38 \\
\hline SSL-PB & 55 & 31 & 40 & 30 \\
\hline Total & 175 & 100 & 130 & 100 \\
\hline
\end{tabular}

implemented vigorously. An attempt was made to compare detection rates before and after the LEC (Table 2).

The detection rate before the campaign was 13 per 100,000 , whereas the rate was $7 / 100,000$ after the campaign. There was an appreciable decline in new case detection including skin smear positive cases after the LEC period.

One of the drawbacks of this campaign was that the non-slum population, especially elite groups, could not be covered satisfactorily, as these people do not allow any physical examination by health workers, either during regular case detection by the trained leprosy workers or during such campaigns, as they prefer to seek private medical consultation. Such elite groups in metropolitan cities may need special approaches, such as involving private physicians and dermatologists to identify possible cases. Though only $70 \%$ of the population could be covered, the striking outcome of this exercise was the detection of nine smear positive cases. The campaign also helped to identify endemic pockets within the project area. Similarly, the whole of Greater Bombay detected 1275 new cases, out of whom 47 (6\%) were skin smear positive MB type. ${ }^{2}$

A large number of migrants from North Indian states like Utter Pradesh, Bihar and Madhya Pradesh enter Bombay slums. Retrospective data analysis of skin smear positive cases recorded in the project area of BLP between 1996 and 1999 showed that 106 (84\%) out of 126 were from outside Bombay. They were mainly from Uttar Pradesh, Madhya Pradesh and Bihar. Thirty $(24 \%)$ patients had BI of $>4+$ and $17(13 \%)$ patients BI $3+$. LECs of smaller scale at regular intervals in selected endemic pockets in urban slum areas will be useful in identifying these imported hidden cases as early as possible and help us in bringing them under MDT.

As the traditional methods of active case finding are not cost effective during the low endemic phase, this cheaper workforce of community volunteers (CV) with appropriate task oriented training can be used to identify leprosy and augment the existing case-finding activities managed by trained manpower, either in the vertical or integrated system. Such campaigns using community volunteers do not replace the existing trained manpower or ongoing leprosy control activities. The deployment of such searchers under the supervision of trained manpower will enable us to reach realistic levels of leprosy elimination in metropolitan areas. All these data show the utility of such massive campaigns in identifying such a large number of cases within a short time and bringing them under treatment to reduce the pool of infection in the community. This should hasten the process of leprosy elimination and to usher in the dawn of a world without leprosy. 
The outcome of LECs in such urban conditions should not be assessed using the strict parameters of other programme assessments. Such campaigns have disadvantages such as over-diagnosis, under-diagnosis (missed cases) and under-coverage of the population, especially of elite groups and working class individuals who are either at their workplaces or on shift duties. However, we have taken all possible precautions to minimize the shortcomings of our LEC as carried out in the population adopted for this study.

\section{References}

1 World Health Organisation. Leprosy elimination campaigns (LECs). Progress during 1997-1998. Weekly Epidemiological Record, 1998; 73: 177-182.

2 Singh MG, Chavan SB. Modified leprosy elimination campaign in Bombay. The mega city. Paper presented at the 15th International Leprosy Congress, Beijing, 7-12 September 1998. 